\title{
Procesos de fiscalización escolar en Chile: Aproximación preliminar al contexto de los establecimientos municipales y particulares subvencionados de la Región Metropolitana
}

\author{
School inspection processes in Chile: A preliminary approach to the context \\ of municipal and subsidized private institutions in the Metropolitan Region
}

\author{
Cristian Oyarzún Maldonado ${ }^{a}$, Rodolfo Israel Soto González ${ }^{b}$ \\ ${ }^{a}$ Facultad de Ciencias Sociales, Universidad de Chile. \\ Correo electrónico: cristian.oyarzunm@usach.cl \\ ${ }^{\mathrm{b}}$ Correo electrónico: rodolfo.soto@usach.cl
}

\begin{abstract}
RESUMEN
La presente investigación explora el desempeño de establecimientos de dos comunas de la Región Metropolitana en Visitas de fiscalización realizadas por la Superintendencia de Educación. Con este fin se analizaron datos de 95 establecimientos en 731 Visitas correspondientes al periodo 2013 - 2015, que fueron comparados según dependencia administrativa y correlacionados con variables como el grupo socioeconómico. Los resultados revelan que la acción fiscalizadora promovió un rápido ajuste a la normativa, pues el desempeño expresado en incumplimientos tiende a equipararse, indistintamente del tipo de dependencia o las características socioeconómicas de los establecimientos. Paralelamente, se distingue que el sector municipal recurre a prácticas irregulares para percibir subvención, a la vez que presenta una débil capacidad para proveer el personal necesario y mejorar las condiciones físicas de sus colegios. Finalmente, el sector particular subvencionado se observa tensionado por las exigencias que regulan las prácticas comerciales indebidas y los mecanismos arbitrarios de exclusión.
\end{abstract}

Palabras claves: fiscalización escolar, accountability escolar, calidad educativa, sistema educacional chileno, sistema de aseguramiento de la calidad.

\section{ABSTRACT}

This research explores the performance of schools in two municipalities of the Metropolitan Region of Chile in Supervising Visits carried out by the Superintendence of Education. For this purpose, data from 731 visits to 95 schools corresponding to the period 2013-2015 were analyzed, compared according to administrative dependence, and correlated with variables such as the socioeconomic status. The results reveal that the supervising action promoted a rapid adjustment to meet regulations, since the performance expressed in instances non-compliance tended to level out, with no distinction between type of dependence or socioeconomic characteristics of schools. At the same time, it was observed that the municipal sector uses irregular practices to receive subsidies, while also having a low ability to provide necessary personnel and to improve the physical conditions of schools. Finally, the private subsidized sector seems to be pressured by the requirements regulating undue commercial practices and arbitrary mechanisms of exclusion.

Key words: school inspection, school accountability, educational quality, Chilean educational system, quality assurance system. 


\section{ANTECEDENTES CONTEXTUALES}

\subsection{MODELO ESCOLAR CHILENO}

A principios de la década de los ochenta, el Estado chileno a través de la dictadura cívicomilitar implementó una política educacional orientada a institucionalizar elementos de mercado y privatización como mecanismos de mejoramiento de la calidad y equidad educativa (Cornejo et al., 2015; Falabella y Opazo, 2014; García-Huidobro, 2007).

Primeramente, esta reforma instaló un sistema de financiamiento basado en un modelo de vouchers o subsidio a la demanda, en que cada establecimiento recibe aportes estatales en función del número de estudiantes que asiste a clases (Cornejo et al., 2015; Elacqua, 2009). En segundo término, impulsó una completa descentralización administrativa de los establecimientos estatales, traspasándolos desde el Ministerio de Educación a las municipalidades. Y en tercer lugar, incentivó la expansión del sector privado en educación con recursos estatales, fijando mínimos requisitos legales para proveer servicios educacionales y creando un mecanismo de financiamiento compartido que les permitió impetrar subvención (Bellei, 2015; Terigi, 2010).

En este contexto, la política pública descrita impulsó una lógica de funcionamiento mercantil en el sistema escolar que, como expone Bellei (2015), se fundamenta en que la competencia desregulada entre establecimientos sería un propulsor del mejoramiento escolar, en la medida que las escuelas con déficit de matrícula no podrían financiarse y, por ende, se encontrarían obligadas a generar acciones dirigidas a optimizar su gestión para atraer a la mayor cantidad de estudiantes.

Respecto de la organización del sistema escolar, este modelo definió que la dependencia administrativa de los establecimientos corresponde a instituciones denominadas sostenedores, municipales o privados, que adquieren ante el Estado la responsabilidad de cumplir con requisitos legales para obtener reconocimiento oficial, junto con recibir y administrar subvención estatal (Darville y Rodríguez, 2007; Terigi, 2010).

De esta forma, según el tipo de entidad sostenedora se establecen cuatro grupos de establecimientos: municipales, particulares subvencionados, particulares pagados y corporaciones de administración delegada (Darville y Rodríguez, 2007). Los establecimientos municipales son financiados por medio de subvención estatal y los particulares subvencionados a través de un financiamiento compartido, constituido por recursos estatales y pagos a cargo de cada familia. Por su parte, el sector particular pagado se sustenta exclusivamente con aportes privados provenientes de las familias. Finalmente, las corporaciones de administración delegada reciben recursos públicos entregados mediante convenios.

La estructura general de este modelo persiste $^{1}$, de manera tal que actualmente la educación escolar chilena es provista a través de un sistema mixto, que cuenta con participación de los sectores público y privado tanto en la producción como en el financiamiento del servicio. En este contexto, diversas investigaciones sostienen que en treinta años de vigencia, el modelo ha generado un proceso de creciente privatización de la

\footnotetext{
La promulgación en 2015 de la Ley de Inclusión № 20.845 elimina el Financiamiento Compartido y modifica aspectos estructurales del sistema, sin embargo, estas modificaciones entraran en vigencia de manera considerablemente gradual, pudiendo extenderse hasta 10 años su plena implementación. Adicionalmente, los nuevos cuerpos legales mantienen íntegramente el Sistema de Aseguramiento de la Calidad.
} 
matrícula y una manifiesta segregación escolar, en el ámbito socioeconómico y académico (Bellei, 2013; Carrasco et al., 2014; Elacqua, 2009; García-Huidobro, 2007; Terigi, 2010).

En este sentido, datos del Mineduc (2015) señalan que entre 2004 y 2014 el sector municipalizado presentó un importante descenso de su matrícula (desde 50\% a 36\%), mientras el sector particular subvencionado experimentó un incremento sustancial de esta variable (desde $41 \%$ a 54\%), siendo la educación particular pagada la única que se mantuvo relativamente estable en este ámbito.

Por su parte, el carácter segregador del modelo se manifiesta a través de una significativa correlación entre grupo socioeconómico y distribución del alumnado en los tres tipos de establecimientos descritos, en donde el sistema municipalizado atiende mayoritariamente a grupos socioeconómicos bajos y solo el particular pagado a aquellos más altos (Bellei, 2013; Carrasco et al., 2014; Elacqua, 2009; García-Huidobro, 2007). A su vez, la estratificación a nivel socioeconómico impactaría en los resultados académicos en la medida que ambas variables también han demostrado correlacionarse significativamente a favor del grupo socioeconómico alto, así por ejemplo lo plantean investigaciones acerca del "efecto par" (Bellei, 2013).

Es así como a contar del año 2000, el modelo escolar chileno se convierte en objeto de críticas desfavorables referidas a sus limitados resultados en términos de calidad y equidad educativa. Sin embargo, como plantea Cornejo et al. (2015), no son evidencias científicas ni discusiones políticas las que promueven cambios en los cimientos organizativos del modelo, sino movilizaciones sociales protagonizadas por estudiantes secundarios desde 2006 en adelante.

La presión social ejercida por el movimiento estudiantil motivó la emergencia de una agenda política en educación escolar, que incluyó la conformación de un Consejo Asesor para la Calidad de la Educación a objeto de elaborar sugerencias técnicas para mejorar la calidad y equidad educativa. El Informe del Consejo Asesor proporcionó diversas recomendaciones que van desde fortalecer el derecho a una educación gratuita y de calidad, hasta reformar la institucionalidad del sistema escolar (Consejo Asesor Presidencial, 2006).

En efecto, el gobierno de la época acogió sugerencias tendientes a transformar la estructura institucional del modelo escolar, propiciando una discusión legislativa que concluyó con la derogación de la Ley Orgánica Constitucional de Enseñanza (Ley N 18.962 , 1990) y la promulgación de dos nuevas leyes, la Ley General de Educación (Ley $\mathrm{N}^{\circ} 20.370$, 2009) y la Ley de Aseguramiento de la Calidad (Ley No 20.529, 2011). Estos cuerpos legales reformulan el rol del Estado como ente garante de una educación de calidad para todos los estudiantes, indistintamente de su origen socioeconómico. Para esto se creó un Sistema de Aseguramiento de la Calidad de la Educación, que incluyó una Superintendencia de Educación, que fiscaliza el cumplimiento de normas y requisitos para proveer educación, y una Agencia de la Calidad, que tiene por objetivo orientar y evaluar los logros de aprendizaje del alumnado como así también los procesos de gestión de los establecimientos.

De esta manera, el Estado formalizó mecanismos para regular -el hasta entonces desregulado-mercado educativo, instaurando un conjunto de estándares de desempeño, metas de resultados y prescripciones legales que deben ser cumplidas por los establecimientos, mediante la permanente evaluación, fiscalización y sanción de posibles transgresiones. Esta política educativa recibe la denominación de "reforma basada en estándares" o "responsabilización por desempeño", en donde un "Estado Evaluador" exige ciertos parámetros de calidad preestablecidos a través de una política de accountability o rendición 
de cuentas externo que las escuelas se comprometen a realizar (Bellei, 2015; Casassus, 2010; Darling-Hammond, 2004; Darville y Rodríguez, 2007; Falabella y Opazo, 2014; Jacob, 2005; Martinic, 2010).

En síntesis, se puede decir que con la implementación del Sistema de Aseguramiento de la Calidad de la Educación, el Estado chileno instaura dispositivos técnicos dirigidos a legitimar la estandarización como instrumento para generar equidad de condiciones en la provisión de servicios educativos, mediante el control y sanción de estándares de aprendizaje y normativas legales que regulan la educación escolar.

\subsection{MODELO DE FISCALIZACIÓN ESCOLAR CHILENO}

La fiscalización refiere a las actividades para evaluar y revisar las acciones de alguna organización considerando su veracidad, razonabilidad y apego a la ley (Adam y Becerril, 1996). Se trata de un mecanismo aplicado principalmente en el sector público y cuyo foco es el control (Melián, 2006). Koontz, Weihrich y Cannice (2008) señalan que el control refiere a la observación y corrección de los comportamientos de individuos y organizaciones para que se ajusten a reglas prescritas.

En Chile, el Sistema de Aseguramiento de la Calidad creó una Superintendencia de Educación que comenzó a funcionar en septiembre de 2012, con el objeto de fiscalizar que los sostenedores de establecimientos educacionales cumplan con la normativa educacional vigente $\mathrm{y}$, a su vez, controlar la legalidad en el uso de los recursos financieros proporcionados mediante subvención estatal (Ley $\left.\mathrm{N}^{\circ} 20.529,2011\right)$.

Este marco legal fue operacionalizado en un sistema público de infracciones, dado a conocer mediante la Resolución Exenta N ${ }^{\circ} 290$ que fija el Modelo de Fiscalización y Estandarización de Hallazgos (Superintendencia de Educación, 2013). El apego a este modelo es exigido a los sostenedores y su observancia es revisada mediante Visitas de fiscalización programadas anualmente, sistemas de rendición de cuentas y también ante denuncias realizadas por miembros de las comunidades escolares. Las contravenciones constatadas durante las Visitas se denominan Hallazgos y son causales de procesos administrativos que eventualmente podrían derivar en sanciones, como multas, amonestaciones, entre otras (Superintendencia de Educación, 2014b).

Las materias fiscalizadas abordan dos ejes temáticos principales, el cumplimiento de la normativa educacional y la adecuada utilización de recursos provenientes de la subvención estatal. En particular, la normativa educacional abarca el conjunto de requisitos prescritos para mantener el reconocimiento oficial, proveer servicios educativos e impetrar subvención, así como también las demás normas legales y operacionales básicas que regulan el funcionamiento de un establecimiento. Por su parte, el uso de recursos estatales es controlado a través de sistemas de rendición de cuentas asociados a distintas subvenciones, como por ejemplo la Subvención Escolar Preferencial (Ley Nº 20.248, 2008).

Entre las condiciones exigidas por la normativa, adquieren preponderancia los ámbitos referidos a la gestión de recursos humanos, infraestructura, mobiliario y equipamiento, pues tienen como finalidad garantizar un ambiente estable y adecuado para el funcionamiento de la organización escolar. Al respecto, se identifican acciones de fiscalización relacionadas directamente con las responsabilidades del sostenedor, tales como verificación de la idoneidad docente del personal, pago de remuneraciones y cotizaciones sociales, constatación de condiciones adecuadas de infraestructura, higiene y seguridad, entre otras. Estos 
aspectos revisten importancia en el logro de resultados académicos, ya que el desempeño y la motivación de las personas que ejercen la labor educativa se encuentra fuertemente asociada a las condiciones laborales en que desarrollan su actividad (Chiavenato, 2009; Murillo, 2011). Asimismo la apropiada provisión de infraestructura, servicios básicos y equipamiento, permite que el personal docente se enfoque exclusivamente en los procesos de enseñanza, evitando la interrupción de las actividades lectivas o destinar esfuerzos para suplir eventuales insuficiencias (Schneider, 2002).

Otro aspecto en que los procesos de fiscalización focalizan su acción, es el cumplimiento de normas dirigidas a cautelar un ambiente escolar seguro, que garantice la buena convivencia, inclusión y participación de todos los estamentos. En este sentido, la normativa exige a cada establecimiento contar con un Manual de Convivencia que regule las relaciones de los actores educativos, además de incorporar protocolos de intervención para situaciones que pongan en riesgo la integridad física y psicológica de los estudiantes (Ley $\left.\mathrm{N}^{\circ} 20.536,2011\right)$. Por su parte, se instauran mecanismos de participación que deben abarcar a la totalidad de la comunidad por medio del funcionamiento efectivo del Consejo Escolar, el Consejo de Profesores y el Centro de Padres (Ley No 20.370, 2009).

De esta forma, la aplicación conjunta del sistema de fiscalización descrito, representa parte de la acción evaluativa del Estado, orientada en este caso a controlar que todos los establecimientos financiados con recursos fiscales dispongan de condiciones suficientes para realizar adecuadamente el proceso educativo y corregir a través de sanciones a aquellos que no satisfagan los estándares preestablecidos.

\section{PROBLEMA DE INVESTIGACIÓN}

La implementación del Sistema de Aseguramiento de Calidad de la Educación Escolar (Ley $\left.\mathrm{N}^{\mathrm{o}} 20.529,2011\right)$ introdujo regulaciones respecto del funcionamiento y resultados esperados en los establecimientos educacionales que disponen de reconocimiento oficial. Estos estándares y normas se encuentran en dos ámbitos principales. Por una parte, refieren a la fiscalización de condiciones suficientes para funcionar dentro de un marco legal preestablecido. Y por otro, aluden a la implementación de un sistema de orientación técnico pedagógico y de evaluación de resultados de aprendizaje.

En un contexto escolar caracterizado por una alta segregación y desigualdad en la provisión de servicios educativos, el Sistema de Aseguramiento releva la estandarización como un medio para lograr equidad en los distintos ámbitos de la calidad educativa, al mismo tiempo que formaliza mecanismos de evaluación y sanción como estímulos que movilizarían el comportamiento de los actores educativos hacia el mejoramiento escolar.

Como ejemplo de lo descrito, el modelo estandarizado de fiscalización aplicado a los sostenedores y sus establecimientos, constituye un marco referencial del nivel de funcionamiento respecto de ciertos requisitos mínimos para la entrega del servicio educativo, que abarcan condiciones suficientes en cuanto a infraestructura, normas de convivencia, planta docente, pago de remuneraciones, uso de recursos estatales, entre otros.

Considerando todo lo antes descrito, la presente investigación pretende explorar el desempeño en Visitas de fiscalización escolar de establecimientos de dos comunas de la Región Metropolitana durante el periodo 2013-2015. En particular, el estudio tiene como objetivos específicos: a) identificar relaciones y diferencias en los incumplimientos 
(Hallazgos) encontrados, en función de características como dependencia, grupo socioeconómico y matrícula; b) comparar los Hallazgos encontrados en los años académicos 2013, 2014 y 2015; y c) caracterizar de acuerdo a una tipología de Hallazgos los establecimientos según dependencia administrativa.

\section{METODOLOGÍA}

\subsection{FUENTES DE INFORMACIÓN}

La información requerida para esta investigación se obtuvo en registros oficiales de servicios públicos del área educación escolar. En particular, se accedió a los datos sobre visitas de fiscalización y hallazgos mediante solicitudes de transparencia ${ }^{2}$ efectuadas a la Superintendencia de Educación. Por otra parte, se utilizaron fuentes de información públicas $^{3}$ del Ministerio de Educación para elaborar bases de datos acerca de grupo socioeconómico, dependencia administrativa y matrícula de los establecimientos.

\subsection{MUESTRA}

La muestra corresponde a 95 establecimientos educacionales subvencionados por el estado, los cuales se encuentran ubicados en dos comunas de la Región Metropolitana de Chile. De este total, 41 cuentan con una dependencia municipal y 54 particular subvencionada, 70 disponen de convenio SEP y 25 no presentan dicho convenio. Asimismo, el nivel socioeconómico promedio de las familias de los estudiantes matriculados en los establecimientos se distribuye de la siguiente forma: 16 en el nivel bajo, 37 en un nivel medio bajo, 23 en el nivel medio, 18 en el nivel medio alto y 1 en el nivel alto. La base de datos utilizada en este estudio contenía los hallazgos de cada establecimiento desde el año 2013 hasta febrero de 2016, agrupándolos en tres años lectivos que comenzaban desde marzo de un año en particular hasta febrero del año siguiente. Esta base de datos contaba con un total de 731 visitas de fiscalización y 1.267 hallazgos encontrados en dichas visitas.

\subsection{VARIABLES}

A objeto de explorar descriptivamente el efecto de los procesos de fiscalización en instituciones escolares, se analizaron las relaciones y diferencias estadísticas entre distintas variables de interés en el contexto del Modelo Escolar Chileno y el Modelo de Fiscalización aplicado. De manera específica, se tomaron en cuenta las siguientes variables:

- Hallazgo ${ }^{4}$ : incumplimiento normativo observado en una visita de fiscalización a un establecimiento educacional, el cual se relaciona directamente con el tipo infraccional (Superintendencia de Educación, 2014a). Con la finalidad de efectuar

\footnotetext{
Acto administrativo establecido en la Ley 20.285 sobre Derecho de Acceso a la Información Pública.

Páginas web: www.agenciaeducacion.cl y www.centrodeestudiosmineduc.cl

Los hallazgos refieren exclusivamente al incumplimiento observado por los fiscalizadores en el momento de la visita, que no necesariamente derivarán en una sanción, pues esto último depende de un proceso administrativo sancionatorio.
} 
comparaciones estadísticas se utilizó la media de hallazgos encontrados por visita de fiscalización. Por otra parte, el análisis de las tipologías de hallazgos se efectuó a partir de categorías temáticas ${ }^{5}$, construidas para la presente investigación.

- Dependencia administrativa: tipo de entidad sostenedora responsable de administrar un establecimiento educacional con reconocimiento oficial. Considerando los objetivos de esta investigación, se emplearon datos de establecimientos de dependencia municipal y particular subvencionada.

- Grupo socioeconómico: conglomerado de características de las familias pertenecientes a un establecimiento educacional que permite su clasificación en nivel bajo, medio bajo, medio, medio alto y alto. Cabe mencionar que esta clasificación se efectúa en función de cuatro variables: nivel educacional de la madre, nivel educacional del padre, ingreso económico total mensual en el hogar e índice de vulnerabilidad (IVE-SINAE).

- Matrícula: cantidad de alumnos inscritos en un establecimiento educacional durante un año lectivo. Para los fines de este estudio se utilizó un promedio de la matrícula de los establecimientos analizados durante el periodo estudiado.

\subsection{ANÁLISIS}

En la presente investigación se realizó un análisis descriptivo de alcance exploratorio, en el cual se establecieron relaciones entre los hallazgos y las demás variables especificadas en el apartado anterior. El diseño utilizado es ex post facto, dado que la información consistía en datos pasados y no se dispuso de manipulación de las variables. Las técnicas de análisis incluyeron estadísticos para correlacionar variables y establecer diferencia de media entre los subgrupos estudiados. Estos procedimientos fueron efectuados por intermedio del paquete estadístico SPSS

\section{RESULTADOS}

\subsection{COMPARACIÓN DE HALLAZGOS EN FUNCIÓN DE OTRAS VARIABLES ESTUDIADAS}

La Tabla 1 muestra las correlaciones entre los hallazgos de los establecimientos con otras variables de interés, como el GSE, el IVE y la matrícula. Además, estas correlaciones se realizaron por tipo de dependencia administrativa. Como es posible observar, únicamente el GSE y el IVE presentan una correlación significativa con la variable hallazgos de todas las organizaciones escolares, siendo esta de carácter débil. Por otro lado, ninguna de las correlaciones por tipo de dependencia es significativa. El análisis de las correlaciones por cada año lectivo da cuenta que únicamente en el 2013 se presenta una correlación significativa de carácter débil entre GSE e IVE con la variable hallazgos, en tanto que en el 2014 y 2015 no se exhibe un nivel de correlación significativa.

Las categorías temáticas de hallazgos se exponen en detalle en la Tabla nº 5 ubicada en la sección resultados. 
Estudios Pedagógicos XLIII, N 3: 225-238, 2017

PROCESOS DE FISCALIZACIÓN ESCOLAR EN CHILE: APROXIMACIÓN PRELIMINAR AL CONTEXTO DE LOS ESTABLECIMIENTOS MUNICIPALES Y PARTICULARES SUBVENCIONADOS DE LA REGIÓN METROPOLITANA

Tabla 1. Correlación entre media de hallazgos por tipo de dependencia y otras variables

\begin{tabular}{|l|c|c|c|}
\hline Tipo de dependencias & GSE & IVE & Matrícula \\
\hline Hallazgos de todos los establecimientos & $-.27 * *$ & $.30 * *$ & -.08 \\
\hline Hallazgos de establecimientos municipales & -.15 & .27 & -.08 \\
\hline Hallazgos de establecimientos particulares subvencionados & -.06 & .11 & -.10 \\
\hline Hallazgos de todos los establecimientos en 2013 & $-.31 * *$ & $.31 * *$ & -.20 \\
\hline Hallazgos de todos los establecimientos en 2014 & -.20 & .21 & -.01 \\
\hline Hallazgos de todos los establecimientos en 2015 & .11 & -.12 & .04 \\
\hline
\end{tabular}

Se empleó Rho de Spearman para todas las correlaciones. ** La correlación es significativa en el nivel 0,01.

Por su parte, en la Tabla 2 se exponen las comparaciones entre los establecimientos municipales y los establecimientos particulares subvencionados considerando distintas variables: hallazgos, GSE, IVE y matrícula. De esta forma, se vislumbra que los establecimientos municipales estudiados tienen significativamente menor GSE, mayor IVE y menor matrícula que los establecimientos particulares subvencionados. En esta misma línea, los hallazgos encontrados en los establecimientos municipales son significativamente mayores a los de establecimientos particulares subvencionados.

Tabla 2. Comparación entre establecimientos municipales y particulares subvencionados en variables estudiadas

\begin{tabular}{|c|c|c|c|c|}
\hline Variable & E. Municipal & E. P. Subvencionado & \multicolumn{2}{|c|}{ Prueba para muestras independientes } \\
\hline & M \pm SD & $\mathbf{M} \pm$ SD & $\mathbf{Z ~ o ~ T}^{*}$ & $\mathbf{P}$ \\
\hline Hallazgos & $1.36 \pm 0.59$ & $0.93 \pm 0.71$ & 3.12 & .000 \\
\hline GSE & $1.78 \pm 0.76$ & $3.02 \pm 0.86$ & -6.04 & .000 \\
\hline IVE & $81.74 \pm 12.69$ & $63.04 \pm 18.54$ & -5.61 & .000 \\
Matrícula & $508.66 \pm 297.70$ & $689.91 \pm 432.79$ & -2.05 & .040 \\
\hline
\end{tabular}

*Se empleó la prueba t de student para la variable hallazgos y para las demás la prueba de Mann-Whitney.

\subsection{COMPARACIÓN DE HALLAZGOS ENTRE AÑOS ESCOLARES}

La Tabla 3 expone una comparación entre los años académicos considerando la variable hallazgos. Como este proceso considera los mismos establecimientos educacionales en los años comparados, se eliminaron del análisis los establecimientos que no contaban con visitas en algún año en particular. De este modo, se constató que los hallazgos en el año escolar 2013 fue significativamente mayor al observado en 2014, mientras que no se presentaron diferencias significativas en la comparación del año académico 2014 con 2015. En contraste, se mostraron diferencias significativas entre el año lectivo 2013 y 2015. Por ende, es posible visualizar 
PROCESOS DE FISCALIZACIÓN ESCOLAR EN CHILE: APROXIMACIÓN PRELIMINAR AL CONTEXTO DE LOS ESTABLECIMIENTOS MUNICIPALES Y PARTICULARES SUBVENCIONADOS DE LA REGIÓN METROPOLITANA

una significativa reducción de los hallazgos en el segundo año académico analizado respecto al primero, manteniéndose este comportamiento hacia el tercer año. Estos resultados son equivalentes si el análisis se hace por tipo de dependencia administrativa de forma separada.

Tabla 3. Comparación de los hallazgos entre años académicos

\begin{tabular}{|c|c|c|c|c|}
\hline Años académico & $\mathbf{1}^{\circ}$ año & $\mathbf{2}^{\circ}$ año & \multicolumn{2}{|c|}{ Prueba para muestras dependientes } \\
\hline & $\mathbf{M} \pm \mathbf{S D}$ & $\mathbf{M} \pm \mathbf{S D}$ & $\mathbf{Z}$ & $\mathbf{P}$ \\
\hline Todos los Establecimientos & & & & .000 \\
\hline $2013-2014$ & $1.86 \pm 1.35$ & $0.93 \pm 0.75$ & -4.52 & .188 \\
\hline $2014-2015$ & $0.88 \pm 0.79$ & $0.65 \pm 0.68$ & -1.32 & .000 \\
\hline $2013-2015$ & $1.89 \pm 1.49$ & $0.64 \pm 0.66$ & -4.79 & \\
\hline E. Municipal & & & & .000 \\
\hline $2013-2014$ & $2.10 \pm 1.25$ & $0.95 \pm 0.65$ & -4.26 & .197 \\
\hline $2014-2015$ & $1.03 \pm 0.76$ & $0.62 \pm 0.80$ & -1.29 & .001 \\
\hline $2013-2015$ & $2.15 \pm 1.35$ & $0.62 \pm 0.80$ & -3.19 & .043 \\
\hline E. P. Subvencionado & & & & .546 \\
\hline $2013-2014$ & $1.56 \pm 1.42$ & $0.92 \pm 0.86$ & -2.02 & .000 \\
\hline $2014-2015$ & $0.80 \pm 0.81$ & $0.67 \pm 0.62$ & -0.60 & \\
\hline $02013-2015$ & $1.75 \pm 1.56$ & $0.65 \pm 0.59$ & -3.65 & \\
\hline
\end{tabular}

Se empleó la prueba de rangos de Wilcoxon.

Por otro lado, la Tabla 4 muestra la comparación de los hallazgos entre establecimientos municipales y particulares subvencionados por año académico. Esta tabla revela que los establecimientos municipales presentan significativamente más hallazgos que los particulares subvencionados únicamente en el año escolar 2013. Tanto en el año 2014 como en el 2015, los establecimientos de ambos tipos de dependencias no presentan diferencias significativas.

Tabla 4. Comparación de los hallazgos entre establecimientos municipales y particulares subvencionados por año académico

\begin{tabular}{|c|c|c|c|c|}
\hline Año académico & E. Municipal & E. P. Subvencionado & \multicolumn{2}{|c|}{ Prueba para muestras independientes } \\
\hline & $\mathbf{M} \pm$ SD & $\mathbf{M} \pm$ SD & $\mathbf{Z}$ & $\mathbf{P}$ \\
\hline 2013 & $2.10 \pm 1.25$ & $1.59 \pm 1.55$ & -2.23 & .026 \\
\hline 2014 & $0.95 \pm 0.65$ & $0.83 \pm 0.84$ & -1.41 & .160 \\
\hline 2015 & $0.62 \pm 0.80$ & $0.62 \pm 0.63$ & -0.60 & .548 \\
\hline
\end{tabular}

Se empleó la prueba de Mann-Whitney. 


\subsection{CARACTERIZACIÓN DE LA TIPOLOGÍA DE HALLAZGOS}

La Tabla 5 muestra la comparación entre establecimientos municipales y particulares subvencionados en función de la clasificación de hallazgos construida. Es así como los establecimientos municipales tienen significativamente más hallazgos que los particulares subvencionados en los siguientes tipos: a) infraestructura, higiene y seguridad, b) participación de la comunidad, c) planta docente y asistentes de la educación, d) registro de asistencia y matrícula y e) servicios asociados a NEE. En contraste, los colegios particulares subvencionados poseen significativamente más de los tipos: a) cobros indebidos, b) financiamiento compartido y c) exclusión del sistema educativo. No presentan diferencias significativas los tipos: a) no registradas, b) pago de cotizaciones y remuneraciones y c) planes de estudio.

Tabla 5. Comparación por tipo de hallazgos entre establecimientos municipales y particulares subvencionados

\begin{tabular}{|l|c|c|c|c|}
\hline Variable & $\begin{array}{c}\text { E. } \\
\text { Municipal }\end{array}$ & $\begin{array}{c}\text { E. P. } \\
\text { Subvencionado }\end{array}$ & \multicolumn{2}{|c|}{$\begin{array}{c}\text { Prueba para muestras } \\
\text { independientes }\end{array}$} \\
\hline Cobros indebidos & M \pm SD & M \pm SD & Z & P \\
Financiamiento compartido & $0.01 \pm 0.03$ & $0.06 \pm 0.10$ & -2.83 & .005 \\
No registradas & $0.01 \pm 0.05$ & $0.03 \pm 0.07$ & -2.53 & .011 \\
Infraestructura, higiene y seguridad & $0.02 \pm 0.04$ & $0.01 \pm 0.03$ & -0.23 & .815 \\
Exclusión del sistema educativo & $0.49 \pm 0.44$ & $0.10 \pm 0.19$ & -6.13 & .000 \\
Participación de la comunidad & $0.01 \pm 0.02$ & $0.05 \pm 0.11$ & -2.26 & .024 \\
Pago de cotizaciones y remuneraciones & $0.04 \pm 0.06$ & $0.02 \pm 0.06$ & -2.30 & .021 \\
Planes de estudio & $0.02 \pm 0.06$ & $0.02 \pm 0.06$ & -0.53 & .594 \\
Planta docente y asistentes de la educación & $0.02 \pm 0.04$ & $0.02 \pm 0.04$ & -0.02 & .980 \\
Registro de asistencia y matrícula & $0.21 \pm 0.14$ & $0.04 \pm 0.07$ & -6.14 & .000 \\
Reglamento interno y normas de convivencia & $0.25 \pm 0.22$ & $0.12 \pm 0.16$ & -3.38 & .001 \\
Rendición de cuentas de subvenciones & $0.04 \pm 0.09$ & $0.07 \pm 0.11$ & -1.88 & .060 \\
Servicios asociados a NEE & $0.12 \pm 0.05$ & $0.14 \pm 0.16$ & -0.25 & .801 \\
\hline
\end{tabular}

Se empleó la prueba de Mann-Whitney. 


\section{DISCUSIÓN Y CONCLUSIONES}

La evidencia presentada en esta investigación da a conocer un efecto coherente con la función fiscalizadora ejercida por la Superintendencia de Educación, en cuanto el comportamiento de los sostenedores y sus establecimientos tiende a ajustarse progresivamente a la normativa educacional vigente, de manera independiente a las características socioeconómicas, el tamaño o el tipo de dependencia administrativa de las organizaciones escolares analizadas. En particular, los resultados generales muestran que desde la implementación de las visitas de fiscalización en 2013, el desempeño global de los establecimientos exhibe una disminución de hallazgos o incumplimientos observados.

En cuanto al grado de correlación existente entre las características socioeconómicas de los establecimientos y su desempeño en visitas de fiscalización, solo en 2013 es factible constatar una relación que describe la presencia de un número mayor de transgresiones a medida que los factores socioeconómicos de las familias son más precarios, no obstante, esta asociación es nula a contar de 2014, tendencia que se mantiene en 2015. Por su parte, el análisis por dependencia administrativa revela que el primer año analizado exhibe un curso de acción distinto a los dos siguientes. En este sentido, las instituciones municipales evidencian una mayor cantidad de infracciones que las particulares subvencionadas, sin embargo, esta diferenciación es suprimida en los años posteriores, lo que da cuenta de una equiparación a partir de la aplicación sistemática de los procesos de fiscalización escolar.

Relacionado con lo anterior, en 2013 se observa un desempeño disímil que distancia a los establecimientos municipales y particulares subvencionados, y que bien podría servir como diagnóstico del comportamiento de los centros educativos cuando la función fiscalizadora residía en la Unidad de Subvenciones del Ministerio de Educación, con atribuciones más restringidas y menores alcances sancionatorios (Leal, 2014). En este contexto, el Sistema de Aseguramiento creó una instancia formal de rendición de cuentas, inexistente hasta ese momento, que tiene en la Superintendencia de Educación facultades legales para promover la responsabilización de las entidades sostenedoras (Darville y Rodríguez, 2007). Al respecto, una interpretación de la evidencia encontrada refiere a la mayor difusión de información respecto del marco normativo y a la presión directa ejercida por los procesos de fiscalización. Estos aspectos son característicos de las políticas de accountability y, en este caso, movilizarían a los proveedores del servicio a cumplir con los parámetros legales preestablecidos.

Aun cuando esta investigación explora el comportamiento de los establecimientos en visitas de fiscalización, también es factible comparar la evidencia obtenida considerando el desempeño en resultados de aprendizaje según tipo de sostenedor. En este sentido, uno de los principales hallazgos de este estudio revela la homologación del desempeño entre dependencias durante el segundo y tercer año, de manera tal que el tipo de administración no constituye una variable importante para explicar el rendimiento global de las unidades educativas ante los requerimientos de la autoridad fiscalizadora. Específicamente, se constata que en el tercer año ambos grupos reportan igual promedio (0.62) de faltas por visita realizada. En concordancia con lo expuesto, diversas investigaciones nacionales describen un desempeño similar de los establecimientos municipales y particulares subvencionados en el ámbito de los aprendizajes, desmitificando creencias populares que suelen posicionar a las entidades particulares por sobre los colegios municipales (Castillo, González y Puga, 2011; Puga, 2011). 
Sin perjuicio de lo antes señalado, el estudio reporta heterogeneidad respecto de la tipología de hallazgos que predominan en cada una de las dependencias. En concreto, las escuelas municipales se caracterizan por presentar incumplimientos en la siguientes categorías: a) infraestructura, higiene y seguridad, b) participación de la comunidad, c) planta docente y asistentes de la educación, d) registro de asistencia y matrícula, y e) servicios asociados a NEE. Cabe mencionar que resulta preocupante la reiteración de faltas en categorías como "infraestructura, higiene y seguridad" y "planta docente y asistentes de la educación", pues evidenciaría una débil capacidad resolutiva del sostenedor respecto de insuficiencias a nivel de las condiciones ambientales y la provisión del personal necesario para desarrollar adecuadamentelos procesos de enseñanza. En el caso de los incumplimientos por irregularidades en registro de asistencia y matrícula, es posible hipotetizar que estas prácticas se originan, en parte, por el afán de generar los recursos necesarios para financiar la producción del servicio. Esto último, podría comprenderse como un efecto del mercado escolar chileno, en la medida que las dinámicas de competencia han mermado la matrícula del sector municipal, lo que disminuye considerablemente sus fuentes de financiamiento.

En lo que respecta a las escuelas particulares subvencionadas, estas presentan más hallazgos en tópicos referidos a: a) cobros indebidos, b) financiamiento compartido y c) exclusión del sistema educativo. Los resultados obtenidos indican que la puesta en marcha de esta política pública deja al descubierto debilidades del mercado educativo en relación a la naturalización de prácticas comerciales y segregadoras. Estos antecedentes, junto a un amplio conjunto de autores que analizan las dinámicas del mercado educacional (Bellei, 2013; Castillo et al., 2011; Elacqua, 2009), develan características inherentes al funcionamiento del sector particular subvencionado, que no necesariamente contribuyen a mejorar la calidad y equidad educativa desde un enfoque de derechos.

Aunque este estudio indaga en un ámbito inexplorado de la política pública educativa, sus resultados deben ser interpretados de manera preliminar en función de algunas limitaciones. Entre estas se puede mencionar que la muestra no representa la diversidad nacional en cuanto al comportamiento de los sostenedores y sus establecimientos, centrándose exclusivamente en la Región Metropolitana. De esta forma, una de las proyecciones alude a complementar la muestra para incorporar múltiples contextos geográficos y sociales, a fin de generalizar patrones nacionales de desempeño en el ámbito estudiado. Por otro lado, si bien se aprecia una rápida asimilación y ajuste a las preescripciones exigidas mediante las visitas de fiscalización, esto no asegura que el desempeño observado se oriente a desplegar acciones genuinas para proteger un bien jurídico o garantizar derechos en el ámbito de la educación escolar. Por ende, futuras investigaciones podrían utilizar metodologías cualitativas a objeto de indagar en la respuesta subjetiva de los actores responsables en relación al sentido y la legitimidad de los procesos de fiscalización.

En conclusión, con independencia de sus limitaciones, el presente artículo constituye un acercamiento inicial al estado actual de una parte de las políticas de "responsabilización por desempeño" asociadas al Sistema de Aseguramiento de la Calidad. Esta aproximación empírica al desempeño de los establecimientos en visitas de fiscalización escolar, reporta evidencia favorable a la acción ejercida mediante la labor fiscalizadora de la Superintendencia de Educación y permite identificar patrones de comportamiento vinculados a cada dependencia administrativa en los territorios comunales estudiados, los que se pueden comprender en el contexto del mercado escolar chileno. 
PROCESOS DE FISCALIZACIÓN ESCOLAR EN CHILE: APROXIMACIÓN PRELIMINAR AL CONTEXTO DE LOS ESTABLECIMIENTOS MUNICIPALES Y PARTICULARES SUBVENCIONADOS DE LA REGIÓN METROPOLITANA

\section{REFERENCIAS BIBLIOGRÁFICAS}

Adam, A. y Becerril, G. (1996). La fiscalización en México. Ciudad de México: Universidad Nacional Autónoma de México.

Bellei, C. (2013). El estudio de la segregación socioeconómica y académica de la educación chilena. Estudios Pedagógicos, 39(1), 325-345. doi:10.4067/S0718-07052013000100019

Bellei, C. (2015). El gran experimento. Mercado y privatización de la educación chilena. Santiago de Chile: LOM Ediciones.

Carrasco, A., Mizala, A., Contreras, D., Santos, H., Elacqua, G., Torche, F., Flores, C. y Valenzuela, J. (2014). Hacia un Sistema Escolar más inclusivo: Cómo reducir la segregación escolar en Chile. Informe de Políticas Públicas $N^{\circ}$ 03. Recuperado el 20 de julio de 2016 desde: http://www. espaciopublico.cl/wp-content/uploads/2016/05/25.pdf.

Casassus, J. (2010). Las reformas basadas en estándares: Un camino equivocado. Revista de Educaçao Educere et Educare, 5(9), 85-107.

Castillo, P., González, A. y Puga, I. (2011). Gestión y efectividad en educación: evidencias comparativas entre establecimientos municipales y particulares subvencionados. Estudios Pedagógicos, 37(1), 187-206. doi:10.4067/S0718-07052011000100010

Chiavenato, I. (2009). Gestión del Talento Humano. Bogotá: McGraw-Hill.

Consejo Asesor Presidencial para la Calidad de la Educación (2006, 11 de diciembre). Informe Final de Consejo Asesor Presidencial para la Calidad de la Educación. Santiago de Chile: Diario Oficial de la República de Chile. Recuperado el 23 de mayo de 2016 desde: http://ciae.uchile.cl/ download.php?file=noticias/824_-1463759466.pdf.

Cornejo, R., Albornoz, N., Castañeda, L., Palacios, D. Etcheberrigaray, G., Fernández, R., Gómez, S., Hidalgo, F. y Lagos, J. I. (2015). Las prescripciones del trabajo docente en el nuevo marco regulatorio de políticas educativas en Chile. Psicoperspectivas, 14(2), 72-83. doi:10.5027/ PSICOPERSPECTIVAS-VOL14-ISSUE2-FULLTEXT-580

Darling-Hammond, L. (2004). Standards, Accountability, and School Reform. Teachers College Record, 106 (6), 1047-1085. doi:10.1111/j.1467-9620.2004.00372.x

Darville, P. y Rodríguez, J. (2007). Institucionalidad, financiamiento y rendición de cuentas en educación. Estudios de Finanzas Públicas de la Dirección de Presupuestos del Ministerio de Hacienda. Recuperado el 14 de marzo de 2016 desde: http://www.dipres.gob.cl/594/ articles-21658_doc_pdf.

Elacqua, G. (2009). The impact of school choice and public policy on segregation: Evidence from Chile. International Journal of Educational Development, 32 (2), 444-453. doi:10.1016/j. ijedudev.2011.08.003

Falabella, A. y Opazo, C. (2014). Sistema de Aseguramiento de la Calidad y procesos de mejoramiento: una mirada desde la gestión educativa. Estudio encargado por el Ministerio de Educación y UNESCO. Recuperado el 17 de enero de 2016 desde: http://biblioteca.uahurtado.cl/ujah/reduc/ $\mathrm{pdf} / \mathrm{pdf} / \mathrm{txt} 1107 . \mathrm{pdf}$

García-Huidobro, J. E. (2007). Desigualdad educativa y segmentación del sistema escolar. Revista Pensamiento Educativo, 40(1), 65-85. Recuperado el 18 de abril de 2016 desde: http:// pensamientoeducativo.uc.cl/index.php/pel/article/download/399/817

Jacob, B. (2005). Accountability, incentives and behavior: the impact of high-stakes testing in the Chicago Public Schools. Journal of Public Economics 89(5), 761-796. doi:10.3386/w8968

Koontz, H., Weihrich, H. y Cannice, M. (2008). Administración. Una perspectiva global y empresarial. Ciudad de México: McGraw-Hill.

Leal, B. (2014). Una mirada histórica sobre la evolución histórica de la inspección escolar en Chile.

Derecho Público Iberoamericano, 5, 87-110. Recuperado el 20 de agosto de 2016 desde: https:// dialnet.unirioja.es/descarga/articulo/5635444.pdf

Ley No 18.962. Ley Orgánica Constitucional de Enseñanza. Diario Oficial de la República de Chile, 
Estudios Pedagógicos XLIII, N 3: 225-238, 2017

PROCESOS DE FISCALIZACIÓN ESCOLAR EN CHILE: APROXIMACIÓN PRELIMINAR AL CONTEXTO DE LOS

ESTABLECIMIENTOS MUNICIPALES Y PARTICULARES SUBVENCIONADOS DE LA REGIÓN METROPOLITANA

Santiago, Chile, 10 de marzo de 1990.

Ley $\mathrm{N}^{\circ}$ 20.248. Ley de Subvención Escolar Preferencial. Diario Oficial de la República de Chile, Santiago, Chile, 1 de febrero de 2008.

Ley $N^{\circ}$ 20.370. Ley General De Educación. Santiago: Diario Oficial de la República de Chile, Santiago, Chile, 12 de septiembre de 2009.

Ley $\mathrm{N}^{\circ}$ 20.529. Sistema Nacional De Aseguramiento de la Calidad De La Educación Parvularia, Básica y Media y Su Fiscalización. Diario Oficial de la República de Chile, Santiago, Chile, 27 de agosto de 2011.

Ley $N^{\circ}$ 20.536. Ley Sobre Violencia Escolar. Diario Oficial de la República de Chile, Santiago, Chile, 17 de septiembre de 2011.

Martinic, S. (2010). Cambios en las Regulaciones del Sistema Educativo ¿Hacia un Estado Evaluador? En S. Martinic y G. Elacqua (Eds.), ¿Fin de ciclo? Cambios en la gobernanza del sistema educativo (pp. 55-78). Santiago: OREAL/UNESCO Santiago y Facultad de Educación, Pontificia Universidad Católica de Chile.

Melián, J. (2006). Relaciones y diferencias entre fiscalización y auditoría. Razones para una reforma de las normas de auditoría del sector público. Auditoría Pública, 39, 33-40.

Recuperado el 20 de agosto de 2016 desde: lapiedad.org.ar/base/Archivos/pg33- 40.pdf

Ministerio de Educación (2015). Centro de Estudios Mineduc. Estadísticas de la Educación 2014. Recuperado el 3 de marzo de 2016 desde: http://www.centroestudios.mineduc.cl/tp_modulos/ tpm_seccion/contVentana.php?cc=2196

Murillo, F. J. (2011). Mejora de la Eficacia Escolar en Iberoamérica. Revista Iberoamericana de Educación, 55, 49-83. Recuperado el 20 de agosto de 2016 desde: rieoei.org/rie55a02.pdf

Puga, I. (2011). Escuela y estratificación social en Chile: ¿cuál es el rol de la municipalización y la educación particular subvencionada en la reproducción de la desigualdad social? Estudios Pedagógicos, 37(1), 213-232. doi:10.4067/S0718-07052011000200013

Schneider, M. (2002). Do school facilities affect academic outcomes? Washington, D.C.: National Clearinghouse for Educational Facilities. Recuperado el 4 de marzo de 2016 desde: http://www. edfacilities.org/pubs/outcomes.pdf.

Superintendencia de Educación (2013). Resolución Exenta $N^{\circ}$ 290: Fija Modelo de Fiscalización y Estandarización de Hallazgos de la Superintendencia de Educación. Recuperado el 26 de febrero de 2016 desde: https://www.supereduc.cl/?categoria-normativa=oficios

Superintendencia de Educación (2014a). Circular $N^{\circ}$ 1: Establecimientos Educacionales subvencionados municipales y particulares. Recuperado el 26 de febrero de 2016 desde: https:// www.supereduc.cl/?categoria-normativa=circulares

Superintendencia de Educación (2014b). Oficio Ordinario $N^{\circ}$ 182: Procesos Administrativos Sancionatorios y Aplicación de Sanciones. Recuperado el 24 de febrero de 2016 desde: https:// www.supereduc.cl/?categoria-normativa=oficios

Terigi, F. (2010). Los sistemas nacionales de inspección y/o supervisión escolar. Revisión de literatura y análisis de casos. Buenos Aires: Instituto Internacional de Planeamiento de la educación IIPEUnesco. 